\title{
Use of hematological parameters as an early screening tool for neonatal sepsis
}

\author{
Bindu Adhikari ${ }^{1}$, Punam Paudyal ${ }^{1}$, Paricha Upadhyaya ${ }^{1}$, Jyoti Agrawal ${ }^{2}$, \\ Basudha Khanal ${ }^{3}$ \\ ${ }^{1}$ Department of Pathology, BP Koirala Institute of Health Sciences, Dharan, Nepal \\ ${ }^{2}$ Department of Pediatrics, BP Koirala Institute of Health Sciences, Dharan, Nepal \\ ${ }^{3}$ Department of Microbiology, BP Koirala Institute of Health Sciences, Dharan, Nepal
}

\author{
Keywords: \\ Blood culture; \\ Hematological scoring \\ system; \\ Neonates; \\ Sepsis;
}

\begin{abstract}
Background: Neonatal sepsis continues to be a major public health problem in developing countries. Due to the nonspecific clinical manifestations of neonatal sepsis, the longer turnaround time for culture reports, an early diagnostic tool is essential for the initiation of appropriate and timely treatment.

Materials and Methods: This is a cross-sectional study of 470 clinically suspected cases of neonatal sepsis admitted in the pediatrics department of B.P. Koirala Institute of Health Sciences, Dharan, Nepal. Following hematological parameters: abnormal total leucocyte count, abnormal total polymorphonuclear count, elevated immature Neutrophils count, elevated immature to total neutrophil ratio, immature to mature neutrophils ratio $\geq 0.3$, platelet count $\leq 150,000 / \mathrm{mm} 3$, and pronounced degenerative changes in Neutrophils were assessed and a score of 1 was assigned to each as per the hematological scoring system of Rodwell. A score $\geq 3$ was considered positive and a score $<3$ was considered negative for the diagnosis of sepsis. Blood culture was taken as a gold standard for diagnosis of neonatal sepsis.
\end{abstract}

Results: Hematological scoring system had fairly high sensitivity, specificity, NPV, and PPV; i.e. $85.96 \%, 84.26 \%, 84.52 \%$, and $85.71 \%$ respectively. The diagnostic accuracy of HSS was high $(85.1 \%)$ in comparison to the maximum diagnostic accuracy of individual hematological parameters, i.e. $74.46 \%$ for the total neutrophil count.

Conclusions: Hematological scoring system can be used as a convenient and early diagnostic tool against neonatal sepsis in developing countries with a high burden of neonatal sepsis because of its high sensitivity and easy availability.

\section{Correspondence:}

Dr. Bindu Adhikari, MD

Department of Pathology, BP Koirala Institute of Health Sciences, Dharan, Nepal

ORCID ID: 0000-0002-8455-8866

Email: bindu.adh7@gmail.com

Received : December $26^{\text {th }} 2020$; Accepted : August $9^{\text {th }} 2021$

Citation: Adhikari B, Paudyal P, Upadhyaya P, Agrawal J, Khanal B. Use of hematological parameters as an early screening tool for neonatal sepsis. J Pathol Nep. 2021;12(2): 1848-53. DOI: 10.3126/jpn. v11i2.33781

Copyright: This is an open-access article distributed under the terms of the Creative Commons Attribution 4.0 International License, which permits unrestricted use, distribution, and reproduction in any medium, provided the original author and source are credited.

\section{INTRODUCTION}

Sepsis refers to the presence of physiological, pathological, and biochemical abnormalities occurring due to dysregulation of the mechanism of the host response to infection. It is a life-threatening situation and can lead to death if treatment is not initiated promptly. ${ }^{1}$ An accepted definition for neonatal sepsis however is lacking, especially in Low and Middle-Income Countries (LMIC) due to resource-poor settings for laboratory evaluations. It is thus recommended that in cases with clinical signs of Possible Serious Bacterial Infection (PSBI), prompt initiation of antibiotics should be done so as to reduce the number of missed cases of sepsis. ${ }^{2}$ On the other hand, neonatal sepsis 
mostly presents with nonspecific clinical manifestations. ${ }^{3}$ Neonatal sepsis is the most common diagnosis in the Neonatal Intensive Care Unit (NICU) and antibiotics are the most commonly used drugs in NICU. If antibiotic treatment is withheld until overt clinical manifestations occur, the outcomes are dismal. However, liberal use of antibiotics can lead to several adverse outcomes including antibiotic resistance. Three out of every 10 cases of deaths due to neonatal sepsis are now caused by resistant pathogens. ${ }^{4}$ The development of an early and simple diagnostic tool would help establish a balance between deaths due to neonatal sepsis and emerging antimicrobial resistance.

Rodwell et al in 1988 utilized the change in a set of simple hematological parameters to establish a hematological scoring system (HSS). ${ }^{5}$ Though several studies have been conducted to test the usefulness of HSS in neonatal sepsis, this scoring system is not yet used very commonly in routine practice. This study was undertaken to establish the usefulness of HSS in neonatal sepsis in the context of a tertiary care center in Nepal.

\section{MATERIALS AND METHODS}

This is a hospital-based cross-sectional study of clinically suspected cases of neonatal sepsis admitted in the pediatrics department of B.P. Koirala Institute of Health Sciences (BPKIHS) from December 2016 to November 2017. Ethical clearance was obtained from Institutional Ethical Review Board, BPKIHS. After taking all aseptic precautions, $2 \mathrm{ml}$ of blood was withdrawn. One $\mathrm{ml}$ of sample was sent for blood culture and sensitivity and the other one $\mathrm{ml}$ of the sample was anticoagulated with EDTA for hematological study. Throughout the study period, a total of 235 cases showed positive blood culture reports, and an equal number of culture-negative cases were thus randomly taken as controls, thereby making the total sample size 470 . For the hematological Study, values of total leucocyte count (TLC) and platelets count were noted using Lab Life D5 Supreme automated hematology analyzer and counterchecked manually. Peripheral blood smears were made and stained with Jenner- Giemsa stain. Differential leucocyte counts (DLC), absolute neutrophil count (ANC), immature neutrophil count (I) (including band form), mature neutrophil count $(\mathrm{M})$ were then performed on stained smears. IT (immature to total neutrophil) ratio and IM (immature to mature neutrophil) ratio were calculated. IT ratio is calculated by dividing the total immature count by total neutrophil count (including both mature and immature neutrophil count). Degenerative changes including Dohle bodies, vacuolization, and toxic granulations were graded as shown in Table 1 and Table 2 respectively. ${ }^{6}$ The hematological scoring system of Rodwell et al was used to calculate the score of each blood sample. (Table 3)

\section{RESULTS}

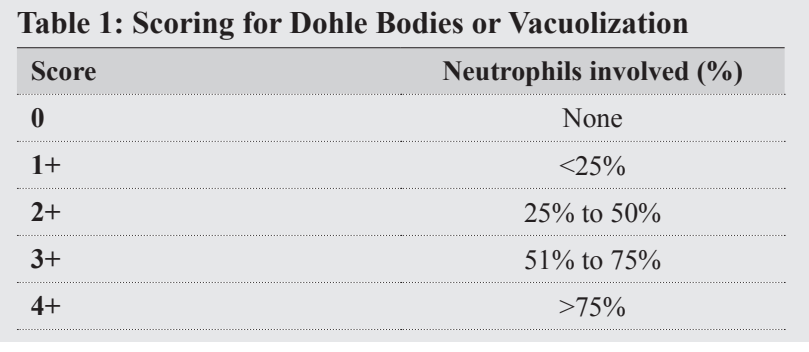

\begin{tabular}{lc}
\multicolumn{2}{l}{ Table 2: Scoring for Toxic Granulations } \\
\hline Score & Interpretation \\
\hline $\mathbf{0}$ & Normal granulation \\
\hline $\mathbf{1 +}$ & Slight \\
\hline $\mathbf{2 +}$ & Dark granules in approximately $50 \%$ neutrophils \\
$\mathbf{3 +}$ & Very heavy granulations in most cells \\
\hline $\mathbf{4}$ & 'Gross' toxic granules causing obscuring of the nucleus \\
\hline
\end{tabular}

\begin{tabular}{lcc}
\multicolumn{3}{l}{ Table 3: Hematological Scoring System (HSS) [6] } \\
\hline Points & Abnormality & Score \\
\hline I: T ratio (>2) & $\uparrow$ & 1 \\
\hline Total PMN count * & $\downarrow$ or $\uparrow$ & 1 \\
I:M ratio & $\geq 0.3$ & 1 \\
Immature PMN Count $\uparrow$ & $\uparrow$ & 1 \\
Total WBC count* & $\downarrow$ or $\uparrow$ & 1 \\
Degenerative changes in PMN & $\geq 3+$ & 1 \\
Platelet count & $\leq 1,50,000 / \mathrm{mm} 3$ & 1
\end{tabular}

$\left(^{*} \leq 5,000 / \mathrm{mm} 3\right.$ or $\geq 25,000 ; 30,000$ and $21,000 / \mathrm{mm} 3$ at birth, 12-24 hrs and day 2 onward respectively)

Of the 470 neonates involved in this study, 253 were male and 217 were female with a male: female ratio of 1.16: 1 . A history of preterm delivery was present in a total of 246 neonates. Out of these, 141 belonged to the culture-positive category and 105 belonged to the culture-negative category. Total 240 neonates had low birth weight (i.e. $<2.5 \mathrm{~kg}$ ), of which 190 belonged to the culture positive and 50 belonged to the culture-negative group. Suspected cases of sepsis were mostly admitted with clinical manifestations of fever, poor feeding, lethargy, irritable cry, difficulty in breathing, diarrhea, convulsion, abdominal distension, hypothermia, or persistent vomiting. Fever and poor feeding were present in a majority of cases, i.e. $60 \%$.

Hematological parameters were assessed in both culturepositive and culture-negative groups. The values that were beyond the normal range and thereby contributed to a score of 1 were taken as 'positive' while those lying within the normal range with zero contribution to the score were taken as 'negative'. Findings are summarized in Table 4. Hematological scores for each sample were then calculated using these hematological parameters. (Table 5)

Also, sensitivity, specificity, PPV and NPV, and accuracy of individual hematological parameters were calculated. 
Table 4: Individual hematological parameters in culture-positive and negative groups

\begin{tabular}{lccc}
\hline Hematological parameter & $\begin{array}{c}\text { Cases with positive } \\
\text { hematological parameter in } \\
\text { Culture positive group (n) }\end{array}$ & $\begin{array}{c}\text { Cases with positive } \\
\text { hematological parameter in } \\
\text { Culture negative group (n) }\end{array}$ & $\begin{array}{c}\text { Total cases with positive } \\
\text { hematological parameters (n) }\end{array}$ \\
\hline TLC & 90 & 22 & 112 \\
Total PMN & 189 & 74 & 263 \\
Immature PMN & 200 & 116 & 316 \\
I:T & 50 & 3 & 53 \\
I:M & 58 & 3 & 61 \\
Degenerative Changes & 146 & 73 & 219 \\
Platelet Counts & 162 & 143 & 305
\end{tabular}

Table 5: Comparison of hematological scoring system with culture results

\begin{tabular}{llcccccc}
\hline Scores & $\mathbf{0}$ & $\mathbf{1}$ & $\mathbf{2}$ & $\mathbf{3}$ & $\mathbf{4}$ & $\mathbf{5}$ & Total \\
Culture positive & 0 & 10 & 37 & 23 & 105 & 60 & 235 \\
Culture negative & 3 & 69 & 133 & 18 & 10 & 2 & 235 \\
Total & 3 & 79 & 170 & 41 & 115 & 62 & 470
\end{tabular}

Table 6: Strength of individual hematological parameters

\begin{tabular}{lccccccc}
\hline Hematologic parameters & Sensitivity (\%) & Specificity (\%) & PPV (\%) & NPV (\%) & Accuracy (\%) & P value \\
Total WBC & 38.3 & 90.6 & 80.36 & 59.50 & 64.46 & $<0.01$ \\
Total PMN & 80.43 & 68.5 & 71.8 & 77.78 & 74.46 & $<0.01$ \\
Immature PMN & 85.11 & 50.64 & 63.29 & 72.27 & 67.87 & $<0.01$ \\
\hline I:T & 21.28 & 98.72 & 94.34 & 55.64 & 60 & $<0.01$ \\
I:M & 24.68 & 98.72 & 95.08 & 56.72 & 61.7 & $<0.01$ \\
Degenerative changes & 62.13 & 91.73 & 66.67 & 90.1 & 65.5 & $<0.01$ \\
Platelet count & 68.94 & 39.15 & 55.76 & 53.11 & 54.04 & 0.06
\end{tabular}

Table 7: Strength of HSS using Score $\geq \mathbf{3}$ as cut off

\begin{tabular}{lcc}
\hline Score & Culture negative & Culture Positive \\
\hline$<3$ & 198 & 33 \\
$\geq \mathbf{3}$ & 37 & 202 \\
Total & 235 & 235 \\
Sensitivity $=85.96 \%$ & \multicolumn{2}{c}{ Specificity $=84.26 \%$} \\
Positive predictive value $=84.52 \%$ & Negative predictive value $=85.71 \%$ \\
Accuracy $=85.10 \%$ & P value $<0.01$
\end{tabular}

(Table 6) The Pearson Chi-square test was applied to calculate the $p$-value.

The results thus highlight the fact when all the hematological parameters are used in combination as in Rodwell's hematological scoring system, the diagnostic accuracy is quite high (85\%) in comparison to the diagnostic accuracy of individual hematological parameters where the highest accuracy (74\%) was of total PMN. HSS can also be used as a useful screening tool against sepsis because of its high sensitivity (86\%) when HSS score $\geq 3$ is used as a cut-off for the presence of sepsis. (Table 7)

\section{DISCUSSION}

Neonatal sepsis continues to be a major public health problem. Each year, around 3 million newborns suffer from sepsis worldwide ${ }^{7}$ leading to around one million deaths per year, the majority of these occurring in developing countries. ${ }^{8}$ Even in developed countries, four out of every ten infants with sepsis either die or experience a major disability such as permanent impairment of neurological development. ${ }^{9}$

In developing countries, infants are more likely to be exposed to several risk factors related to neonatal sepsis. Different studies have shown that factors like prolonged rupture of membrane (PROM), prematurity ${ }^{3}$, low Apgar score at birth ${ }^{10}$, and $\mathrm{LBW}^{11}$ are linked with the incidence of sepsis. In our study, a history of preterm delivery was present in $246(52 \%)$ of total neonates. Out of these, 141 belonged to the culture-positive category and 105 belonged to the culture-negative category. Total 240 neonates had a low birth weight of which 190 belonged to the culture positive and 50 belonged to the culture-negative group. Preterm and low birth weight babies have lower levels of immunoglobulin and less developed immune systems thereby being more susceptible to sepsis with a higher risk of mortality and lifelong neurodevelopmental handicap. ${ }^{12}$ However, the accuracy of the pediatric consensus definitions has not yet been assessed in preterm infants, and using these criteria is untenable because of several limitations related to 
the developmental maturity of preterm infants. ${ }^{12}$ In a study by Adatara et al, no relation was seen between neonatal sepsis and gestational age, birth weight. ${ }^{10}$

The incidence of sepsis was higher $(53.82 \%)$ in male neonates than in female neonates $(46.1 \%)$ in our study. This finding is consistent with that of other studies. ${ }^{13-16}$ In this study, neonates mostly presented with chief complaints of poor feeding $(60 \%)$ and fever $(60 \%)$. Studies have suggested that the clinical presentation of neonatal sepsis is quite nonspecific ${ }^{3,16}$ and thus clinicians need to be more alert while handling these cases.

Blood culture is the gold standard test for the diagnosis of neonatal sepsis. ${ }^{17}$ Factors like the small blood volumes obtained from neonates, maternal intrapartum antimicrobial exposure, and the presence of low or intermittent bacteremia can yield false-negative culture reports. ${ }^{17}$ With the increased understanding of pathogenesis and immune mechanisms involved in neonatal sepsis, several newer inflammatory markers such as interleukin-6, interleukin-8, plasma elastase, neutrophil CD64 have been known to be useful in the diagnosis of sepsis. These markers are highly sensitive and specific, but they require expensive and sophisticated technologies, not easily affordable by developing countries. ${ }^{18,19}$ On the other hand, in middleincome countries, the incidence rate of neonatal sepsis is nearly 40 times higher than in high-income countries and the mortality rate is two times. ${ }^{7}$

HSS utilizes parameters that can be assessed quickly and used as an early diagnostic tool for neonatal sepsis especially in developing countries where the burden of neonatal sepsis is higher and sophisticated labs are lacking at many places. Due to the insensitivity of physical examination and culture, even the modern diagnostic approaches for neonatal sepsis have chosen to use many of the parameters that are a part of HSS in combination with the acute phase reactants ${ }^{17}$. The different parameters used in the HSS have individual strengths and weaknesses ${ }^{13,14,19-22}$. The HSS uses them in combination to improve the accuracy of diagnosis of neonatal sepsis. Studies conducted on HSS to date have come to different conclusions regarding the strength of individual parameters used in the HSS. Most of these studies showed that these parameters are more reliable in the detection of sepsis when used in combination as HSS rather than individually. ${ }^{13,14,23,24} \mathrm{~A}$ comparison between the strength of HSS as calculated by different studies has been shown in Table 8.

Apart from these, few other studies have also analyzed the strength of individual parameters used in HSS. Abnormal immature to total neutrophil ratio followed by an abnormal immature to mature neutrophil ratio was found to be the most sensitive indicators for the diagnosis of neonatal sepsis in the studies conducted by Ghosh et al, Narasimha et al and Majumdar et al. ${ }^{28-30}$ In the study by Priyanka et al, absolute neutrophil count and the increased immature neutrophil count had the highest specificity of $91 \%$ and $92 \%$ respectively. ${ }^{31}$

The HSS may also be used with newer diagnostic modalities when possible to further increase the accuracy. In a study by Khair et al in 2012, HSS score $\geq 4$ had a sensitivity of $100 \%$ and specificity of $60 \%$. C-reactive protein (CRP) had a sensitivity of $75 \%$ and specificity of $74 \%$. The combination of score $\geq 4$ and CRP had a sensitivity of $75 \%$, specificity $85 \%$, positive predictive value (PPV) $41 \%$, and negative predictive value (NPV) $96 \%$. This combination was thus useful to differentiate the septicemic from nonsepticemic neonates and thereby help in the judicious use of antibiotics. ${ }^{32}$

In our study when the hematological score $\geq 3$ was used as a positive cut-off for the diagnosis of neonatal sepsis, diagnostic accuracy was high. Saleem et al and Yusuf et al have also used this cut-off in their studies and thus found the sensitivity of HSS to be $71 \%$ and $90 \%$ respectively while the specificity was $72 \%$ and $74.5 \%$ respectively. ${ }^{14,21}$ Whereas some other studies have used score $\geq 4$ as their diagnostic cut off thereby leading to a maximum sensitivity of $100 \%$ and specificity of $86 \% .{ }^{23}$ Majumdar et al have concluded in their study that though the score $\geq 3$ is highly sensitive, score $\geq 4$ has a higher specificity and PPV and hence can be considered as the more reliable screening tool for the diagnosis of sepsis ${ }^{30}$. Studies have found that the certainty of sepsis being present increases with the increase in the score. ${ }^{26,28}$

An ideal screening test should have high sensitivity and negative predictive value that would enable it to detect all the patients with the disease and exclude all those cases without it. ${ }^{26}$ HSS appears to have fulfilled these criteria in all the studies conducted so far and hence can be taken as an effective screening tool against neonatal sepsis.

\section{CONCLUSIONS}

When all the hematological parameters are used in combination as in Rodwell's HSS, the diagnostic accuracy is quite high in comparison to the diagnostic accuracy of individual hematological parameters. Because of its high sensitivity when HSS score $\geq 3$ is used as a cut-off for the presence of sepsis, HSS can be used as a useful screening tool against neonatal sepsis. This test can be quickly assessed even in resource-poor setups and hence suitable for use in low-income countries with a greater burden of neonatal sepsis than their developed counterparts.

\section{Conflict of Interest: None}

\section{REFERENCES}


1. Singer M, Deutschman CS, Seymour CW, et al. The Third International Consensus Definitions for Sepsis and Septic Shock (Sepsis-3). JAMA. 2016;315(8):801810. $\underline{\text { Crossref }}$

2. Fuchs A, Bielicki J, Mathur S, Sharland M, Van Den Anker JN. Reviewing the WHO guidelines for antibiotic use for sepsis in neonates and children. Paediatr Int Child Health. 2018;38(sup1):S3-S15. Crossref

3. Singh M, Gray CP. Neonatal Sepsis. [Updated 2019 Nov 25]. In: StatPearls [Internet]. Treasure Island (FL): StatPearls Publishing; 2020. Website

4. Laxminarayan R, Matsoso P, Pant S, et al. Access to effective antimicrobials: a worldwide challenge. Lancet 2016; 387(10014):168-75 $\underline{\text { Crossref }}$

5. Rodwell RL, Leslie AL, and Tudehope DI. Early Diagnosis of Neonatal Sepsis using a Hematologic Scoring System. The Journal of Pediatrics 1988;112(5):761-7. $\underline{\text { Crossref }}$

6. Zipursky A, Palko J, Milner R, Akenzua GI. The hematology of bacterial infection in premature infants. Pediatrics 1976;57 (6) 839-53 Website

7. Fleischmann-Struzek C, Goldfarb DM, Schlattmann P, Schlapbach LJ, Reinhart K, Kissoon N. The global burden of paediatric and neonatal sepsis: a systematic review. The Lancet Respiratory medicine 2018; 6(3): 223-30. Crossref

8. Getabelew A, Aman M, Fantaye E, Yeheyis T. Prevalence of Neonatal Sepsis and Associated Factors among Neonates in Neonatal Intensive Care Unit at Selected Governmental Hospitals in Shashemene Town, Oromia Regional State, Ethiopia, 2017. International Journal of Pediatrics 2018. Article ID 7801272 Crossref

9. Wynn JL. Defining neonatal sepsis. Curr Opin Pediatr. 2016;28(2):135-40. Crossref

10. Adatara P, Afaya A, Salia SM, et al. The Scientific World Journal. Vol 2019. Article ID 9369051. Crossref

11. Rugolo LMSS, Bentlin MR, Mussi-Pinhata M, et al. Late-Onset Sepsis in very Low Birth Weight Infants: A Brazilian Neonatal Research Network Study, Journal of Tropical Pediatrics 2014;60:415-21, $\underline{\text { Crossref }}$

12. Goldstein B, Giroir B, Randolph A. International pediatric sepsis consensus conference: definitions for sepsis and organ dysfunction in pediatrics. Pediatr Crit Care Med. 2005;6(1):2-8. $\underline{\text { Crossref }}$
13. Khair K, Rahman M, Sultana $T$ et al. Role of Hematologic Scoring System in Early Diagnosis of Neonatal Septicemia. Bangabandhu Sheikh Mujib Medical University Journal, 2010;3(2), 62-67. Website

14. Arif S, Ehsan A, Arif M, Hussain J, Bano R. Early Diagnosis of Neonatal Sepsis Through Hematological and Biochemical Markers. Gomal Journal of Medical Sciences. 2012;58(2):178-82. Website

15. Saleem M, Shah KI, Cheema SM, Azam M. Hematological Scoring System for Early Diagnosis of Neonatal Sepsis. Journal of Rawalpindi Medical College. 2014; 18(1): 68-72. Website

16. Meirina F, Lubis B, Sembiring $T$, Rosdiana N, Siregar O. Hematological Scoring System as an Early Diagnostic Tool for Neonatal Sepsis. Paediatr Indones. 2015; 55(6): 315-321 Website

17. Iroh Tam P, Bendel C. Diagnostics for neonatal sepsis: current approaches and future directions. Pediatr Res 2017;82:574-83. . rossref

18. Streimish I, Bizzarro M, Northrup V, et al. Neutrophil CD64 as a diagnostic marker in neonatal sepsis. Pediatr Infect Dis J. 2012;31(7):777-81. Crossref

19. Vinay BS, Girish GN, Adhikari S, Hugara S. Evaluation of Septic Screen as a Diagnostic Tool for Neonatal Sepsis in a Tertiary Hospital at Mysore. Sch. J. App. Med. Sci.2015; 3(2G):1005-10. Website

20. Bhandari V, Wang C, Rinder C, Rinder H. Hematologic profile of sepsis in neonates: neutrophil CD64 as a diagnostic marker. Pediatrics. 2008;121(1):129-34. $\underline{\text { Crossref }}$

21. Yusuf MM, Yasmeen BHN, Chowdhury, MA KA. Evaluation of Hematological Scoring System (HSS) for Early Diagnosis of Neonatal Sepsis. Northern International Medical College Journal. 2016; 7 (2): 135-8 Website

22. Kayode-Adedeji BO. Early Diagnosis of Neonatal Sepsis: A Review of the Current Methods in Clinical Practice. Asian Journal of Medicine and Health. 2017; 4(2): 19. Website

23. Shirazi H, Riaz S, Tahir R. Role of the Hematological Profile in Early Diagnosis of Neonatal Sepsis. Ann. Pak. Inst. Med. Sci 2010; 6(3):152-6. Website

24. Makkar M, Gupta C, Pathak R, Garg S, Mahajan NC.Performance Evaluation of Hematologic Scoring 
System in Early Diagnosis of Neonatal Sepsis. Journal of Clinical Neonatology. 2013; 2(1), pp.25-29. Website

25. Ramamurthy BD, Shivashekar G, Balaji R. Haematological scoring system: Can it be used as a routine screening tool in early diagnosis of neonatal sepsis? International Journal of Clinical and Diagnostic Pathology 2019; 2(1):10-3. Crossref

26. Nayak R, Subhan AR, Nisha TG. Diagnostic utility of haematological parameters and $\mathrm{C}$-reactive protein in early detection of neonatal septicaemia in developing countries. Indian Journal of Pathology and Oncology, January-March, 2018;5(1):112-7. Website

27. Bhalodia MJ, Hippargi SB, Patil MM. Role of hematological scoring system in diagnosis of neonatal sepsis. J Clin Neonatol 2017;6:144-7. Website

28. Ghosh S, Mittal M, Jaganathan G. Early diagnosis of neonatal sepsis using a hematological scoring system. Indian J Med Sci. 2001;55(9):495-500. Website
29. Narasimha, A and Harendra Kumar, M.L. Significance of Hematological Scoring System (HSS) in Early Diagnosis of Neonatal Sepsis. Indian J Hematol Blood Transfus 2011;27(1):14-7. Crossref

30. Majumdar A, Jana A, Jana A, Biswas S, Bhattacharyya S. Hematologic Scoring System: A guide to decide judicious use of antibiotics in neonatal septicemia in developing countries. Journal of Applied Hematology. 2013; 4(3):110-3. Website

31. Priyanka T, Hemalata. Basic Hematological Scoring System-Is it the most Accurate Neonatal Sepsis Predictor? National Journal of Laboratory Medicine. 2018;7(3): 29-33. Website

32. Khair KB, Rahman MA, Sultana T, Roy CK, Rahman MQ, Ahmed AN. Early diagnosis of neonatal septicemia by hematologic scoring system, C-reactive protein and serum haptoglobin. Mymensingh Med J. 2012;21(1):85-92. Website 